\title{
AUTORES CITADOS EN LOS TRABAJOS ESPAÑOLES DE CIRUGÍA DIGESTIVA (1991-1995)
}

\author{
Josb Miguel Sisez Gómez"; Diana Gómez Torradillos*;; Pablo Ramírez \\ Romero"tht; Manuel Valora Candel*
}

Resumen: Para identificar las influencias cientificas ejercidas sobre los autores españoles en cirugia digestiva, se analizaron las referencias contenidas en los 483 artículos de cirugfa publicados en Revista Española de Enfermedades Digestivas entre 1991 y 1995. Los autores citados fuéron distribuidos en orden decreciente del número de citas recibidas y esta distribución fue comparada con la térica de Lotka y con la obtenida cuando se considera exclusivamente al primer firmante del artículo citado. Hemos obtenido 23.326 citas a $\mathbf{1 5 . 8 8 6}$ autores distintos, procedentes de $\mathbf{8 . 1 2 5}$ referencias. La distribución de autores y citas por autor se ajusta a la expresión $A c=13931 \mathrm{c}^{-2,9}$, y las citas a primeros firmantes a la expresión $A c=6515,8 \mathrm{c}^{-3.23}$; ambas indican una alta dispersión de citas entre autores escasamente influyentes. Se confirma la elevada influencia de los autores españoles, ya que son de esta nacionalidad el más citado, tres de los cinco autores más citados y seis de los 20 más citados. De estos 20 autores, siete (uno español) no colaboran con el resto, y los otros 13 se organizan en cinco equipos de trabajo, dos de ellos españoles.

Palabras clave: cirugía digestiva, bibliometría, análisis de citas, citas a autores.

Summary: The references contained in the 483 surgical papers published by Revista Espaniola de Enfermedades Digestivas (1991-1995) were analysed with the aim of identifying the scientific influences exercised on Spanish authors in digestive surgery. The authors cited were distributed in decreasing order of the number of citations. This distribution was compared with the theoretical one postulated by Lotka, and with that obtained when only the first author of the cited papers was considered. 23,326 citations to 15,886 different authors were taken from 8,125 references. The distribution of the cited authors and the number of citations per author follows the expression $A c=13931 c^{-2.9}$ and the citations to the first author of the cited papers to $A c=6515.8 c^{-3.23}$. Both expressions show that there exists a wide spread of citations in authors with little influence. The high influence of Spanish authors on themselves is confirmed: the most cited author is a Spaniard, as are 3 of the 5 most cited authors and 6 of the 20 most cited authors. Seven (one of them Spanish) of these 20 authors don't have collaboration with the rest, and the other 13 are organised in five scientific teams, two of which are Spanish.

Key words: digestive surgery, bibliometrics, citation analysis, citations to authors.

\footnotetext{
* U.D. de Historia de la Medicina y Documentación Médica. Universidad de Murcia. Correo-e: jmsaez@fcu.um.es.

** Departamento de Cirugía. Universidad de Murcia.

*** Servicio de Cirugía. Hospital Universitario Virgen de la Arrixaca. Murcia.

Recibido: 5-10-98. Segunda versión: 2-6-99.
} 


\section{Introducción}

Las referencias contenidas en los artículos publicados en un período concreto de tiempo y en una determinada área de trabajo, constituyen lo que se ha llamado «literatura activa circulante» (1), es decir, la literatura que contiene la información viva que se está utilizando en ese momento.

El análisis de las referencias contenidas en los trabajos de la cirugía digestiva en España es un elemento indispensable para conocer y estudiar el consumo de la información científica por parte de la comunidad de especialistas en nuestro país, así como para la obtención de indicadores de repercusión o influencia que los diferentes autores tienen sobre su actividad (2).

El presente trabajo parte del análisis de las referencias contenidas en los artículos de tema quirúrgico publicados por la revista que los especialistas consideran como más importante de nuestro país en patología digestiva, la Revista Española de Enfermedades Digestivas, con el fin de identificar a los autores más influyentes en la especialidad. Partimos de la hipótesis de que la influencia de los autores españoles es mayor de lo que habitualmente se supone $(3,4)$ y por supuesto, muy diferente de lo que reflejan los repertorios de citas al uso.

\section{Material y métodos}

De los trabajos con contenido científico de cualquier tipo documental (originales, cartas, notas clínicas) publicados en Revista Española de Enfermedades Digestivas, se han seleccionado todos los de contenido quirúrgico aparecidos entre 1991 y 1995. De éstos, se han obtenido todos los autores de las referencias contenidas en los mismos y se han clasificado por orden decreciente de citas recibidas. Esta distribucion se ha comparado con la obtenida al considerar tan sólo al primer firmante de las referencias, y se ha analizado la concordancia entre ambas distribuciones y el modelo propuesto por Lotka para el análisis de la productividad (5). Esta aplicación concreta supone que, dada la gran diferencia que suele observarse entre los escasos autores que concentran un gran número de citas y los numerosos que reciben una o pocas citas, debe existir una relación directa entre el logaritmo del número de citas por autor y el logaritmo del número de autores que recibe exactamente ese número de citas (6), de acuerdo con la ecuación $A c=A_{1} c^{-2}$, donde $A_{1}$ es el número de autores que recibe una sola cita, $c$ el número de citas por autor y Ac el número de autores que recibe exactamente «c» citas.

\section{Resultados}

Se han analizado (tabla 1) las 8.125 referencias contenidas en los 483 artículos de tema quirúrgico publicados por Revista Española de Enfermedades Digestivas entre 1991 y 1995 , lo que supone una cuarta parte de la producción de la revista y el $100 \%$ de su producción quirúrgica. La media de referencias por trabajo es de 16,8 .

Las referencias contenían (tabla 2) 23.326 citas de 15.886 autores distintos (1,5 citas/autor y 2,87 autores/referencia, si bien en 1.454 referencias se incluyen las partí- 
culas «et al» 0 «y col», indicadoras de que existen firmas en las referencias que no han sido identificadas por el autor citador y en $\mathbf{4 6}$ referencias no puede identificarse a los autores.

TABLA 1

Producción quirúrgica en Revista Española de Enfermedades Digestivas

\begin{tabular}{|c|c|c|c|c|c|}
\hline Afio & Articulos & $\begin{array}{c}\text { Articulos } \\
\text { cirugia }\end{array}$ & Porcentaje & $\begin{array}{c}\text { Numero } \\
\text { referencias }\end{array}$ & $\begin{array}{c}\text { Referencias } \\
\text { trabajo }\end{array}$ \\
\hline 1991 & 336 & 99 & 29,46 & 1.564 & 15,80 \\
1992 & 316 & 99 & 31,33 & 2.050 & 20,71 \\
1993 & 395 & 103 & 26,07 & 1.923 & 18,67 \\
1994 & 462 & 100 & 21,64 & 1.822 & 18,22 \\
1995 & 459 & 82 & 17,86 & 766 & 9,34 \\
Total & 1968 & 483 & 24,54 & .8 .125 & 16,82 \\
\hline
\end{tabular}

Tabla 2

Citas al conjunto de autores y a los primeros firmantes de las referencias

\begin{tabular}{|c|c|c|c|c|c|}
\hline \multicolumn{3}{|c|}{ Citas a todos los autores } & \multicolumn{3}{|c|}{ Citas a primeros firmantes } \\
\hline $\begin{array}{l}\text { Citas/ } \\
\text { autor }\end{array}$ & $\begin{array}{l}\text { Número } \\
\text { autores }\end{array}$ & $\begin{array}{l}\text { Número } \\
\text { citas }\end{array}$ & $\begin{array}{l}\text { Citas/ } \\
\text { autor }\end{array}$ & $\begin{array}{l}\text { Número } \\
\text { autores }\end{array}$ & $\begin{array}{l}\text { Número } \\
\text { citas }\end{array}$ \\
\hline $\begin{array}{c}1 \\
2 \\
3 \\
4 \\
5 \\
6 \\
7 \\
8 \\
9 \\
10 \\
11 \\
12 \\
13 \\
14 \\
15 \\
16 \\
17 \\
18 \\
19 \\
20 \\
23 \\
36 \\
40 \\
\text { Total }\end{array}$ & $\begin{array}{r}12.190 \\
2.192 \\
733 \\
341 \\
164 \\
89 \\
44 \\
36 \\
27 \\
16 \\
14 \\
8 \\
7 \\
5 \\
5 \\
5 \\
3 \\
2 \\
1 \\
1 \\
1 \\
1 \\
1 \\
15886\end{array}$ & $\begin{array}{r}12.190 \\
4.384 \\
2.199 \\
1.364 \\
820 \\
534 \\
308 \\
288 \\
243 \\
160 \\
154 \\
96 \\
91 \\
70 \\
75 \\
80 \\
51 \\
36 \\
19 \\
20 \\
23 \\
36 \\
40 \\
23.6\end{array}$ & $\begin{array}{r}1 \\
2 \\
3 \\
4 \\
5 \\
6 \\
7 \\
8 \\
9 \\
10 \\
11 \\
13 \\
18\end{array}$ & $\begin{array}{r}4.896 \\
735 \\
226 \\
99 \\
43 \\
22 \\
11 \\
11 \\
6 \\
2 \\
2 \\
1 \\
1\end{array}$ & $\begin{array}{r}4.896 \\
1.470 \\
678 \\
396 \\
215 \\
132 \\
77 \\
88 \\
54 \\
20 \\
22 \\
13 \\
18\end{array}$ \\
\hline \multicolumn{3}{|c|}{$\begin{array}{c}A c=13.931 c^{-2.9} \\
r=-0,98, p<0,001\end{array}$} & \multicolumn{3}{|c|}{$\begin{array}{c}A c=6.515,8 c^{-3,23} \\
r=-0,99, p<0,001\end{array}$} \\
\hline
\end{tabular}


Distribuidos los autores firmantes de las referencias en orden decreciente del número de citas recibidas (tabla 2), se obtienen como valores extremos un solo autor con 40 citas y 12.190 autores que tan sólo reciben una cita.

$\mathrm{Si}$ se considera exclusivamente a los primeros firmantes de las referencias se obtiene una distribución muy diferente: un solo autor con 18 citas y 4.896 autores con una sola cita. La relación de los autores y primeros firmantes más citados se ofrece en las tablas 3 y 4 .

Tabla 3

\section{Autores más citados en las referencias}

\begin{tabular}{|c|c|}
\hline Citas & Autor \\
\hline $\begin{array}{l}40 \\
36 \\
23\end{array}$ & $\begin{array}{l}\text { Parrilla, P. Serv. Cirugía. Hosp Univ. Virgen de la Arrixaca. Murcia. España } \\
\text { Starlz, T. E. Dpt. Surgery. Univ. Pittsburgh. Pennsylvania. USA } \\
\text { Cameron, J. L. Dpt. Surgery. Johns Hopkins University School of Medicine. Baltimore. Maryland. } \\
\text { USA }\end{array}$ \\
\hline $\begin{array}{l}20 \\
19\end{array}$ & $\begin{array}{l}\text { Pera Madrazo, C. Serv. Cirugía. Hosp Univ. Reina Soffa. Cordoba. España. } \\
\text { Garcia Alonso, I. Serv. Cirugía y Lab. Cir. Experimental. Hospital de Basurto. Univ. del País Vasco. } \\
\text { España }\end{array}$ \\
\hline 18 & $\begin{array}{l}\text { Williams, R. Inst. of Liver Studies. King's College Hospital and King's College School of Medicine, } \\
\text { London, UK }\end{array}$ \\
\hline 18 & Bloom, S. R. Royal Postgraduate Medical School. Hammersmith Hospital. London. UK \\
\hline 17 & Mendez, J. Serv. Cirugia y Lab. Cir. Experimental. Hosp. Basurto, Univ. País Vasco. España \\
\hline 17 & Polak, J. M. Royal Postgraduate Medical School. Hammersmith Hospital. London. UK \\
\hline 17 & $\begin{array}{l}\text { Tzakis, A. G. Dpt. Surgery. Transplantation Institute. Univ. Pittsburgh Medical Center. Pennsylvania. } \\
\text { USA }\end{array}$ \\
\hline 16 & Hogan, W. J. Medical College of Wisconsin. Milwaukee, USA \\
\hline 16 & Iwatsuki, S. Dpt. Surgery. Univ. Pittsburgh School of Medicine. Pennsylvania. USA. \\
\hline 16 & Morson, B. C. Dpt. Pathology. Free University Hospital of Amsterdam. The Netherlands. \\
\hline 16 & Sánchez Bueno, F. Serv. Cirugía. Hosp. Univ. Virgen de la Arrixaca. Murcia. Espanfa. \\
\hline 16 & Thompson, J. Texas Medical Branch. Galveston. USA \\
\hline 15 & Calne, R. Y. Dpt. Surgery. University of Cambridge Clinical School, UK \\
\hline 15 & De Meester, T. Dpt. Surgery. University of Southern California School of Medicine. Los Angeles. USA \\
\hline 15 & Serv. Chirurgie Generale. Hopital International de l'Université de Pari \\
\hline 15 & Ser. Cirugía. Hosp \\
\hline 15 & Todo, S. Dpt. Surgery. Transplantation Institute. Univ. Pittsburgh Medical Center. Pennsylvania. \\
\hline
\end{tabular}

Tabla 4

Autores más citados como primeros firmantes de las referencias

\begin{tabular}{|c|l|c|l|}
\hline Citas & \multicolumn{1}{|c|}{ Autor } & Citas & \multicolumn{1}{c|}{ Autor } \\
\hline 18 & Starlz TE & 9 & Pera Madrazo C \\
13 & Garcí Alonso & 9 & Burke A \\
11 & Bismuth H & 9 & Cuscheri \\
11 & Cotton P & 8 & Ellis F \\
10 & Calne RY & 8 & Hebrero San Martín J \\
10 & Dubois F & 8 & Law W \\
9 & Blumberg N & 8 & Martínez Ramos \\
9 & Dowling R & 8 & Moreira \\
9 & Guite & 8 & Ramirez Romero P \\
9 & Lankisch P & 8 & Reddick E \\
9 & Parrilla P & 8 & Sanfey H \\
& & 8 & Washaw \\
\hline
\end{tabular}


Figura 1

Distribución del número de citas por autor.

En línea continua se presentan las distribuciones de citas observadas entre todos los autores $\left(A c=13.931 c^{-2.9}\right)$ y entre primeros firmantes $\left(A c=6.515,8 c^{-3.23}\right)$.

En línea discontinua las correspondientes a las distribuciones teoricas de citas entre todos los autores $\left(\mathrm{Ac}=12.190 \mathrm{c}^{-2}\right)$ y entre los primeros firmantes $\left(\mathrm{Ac}=4.896 \mathrm{c}^{-2}\right)$

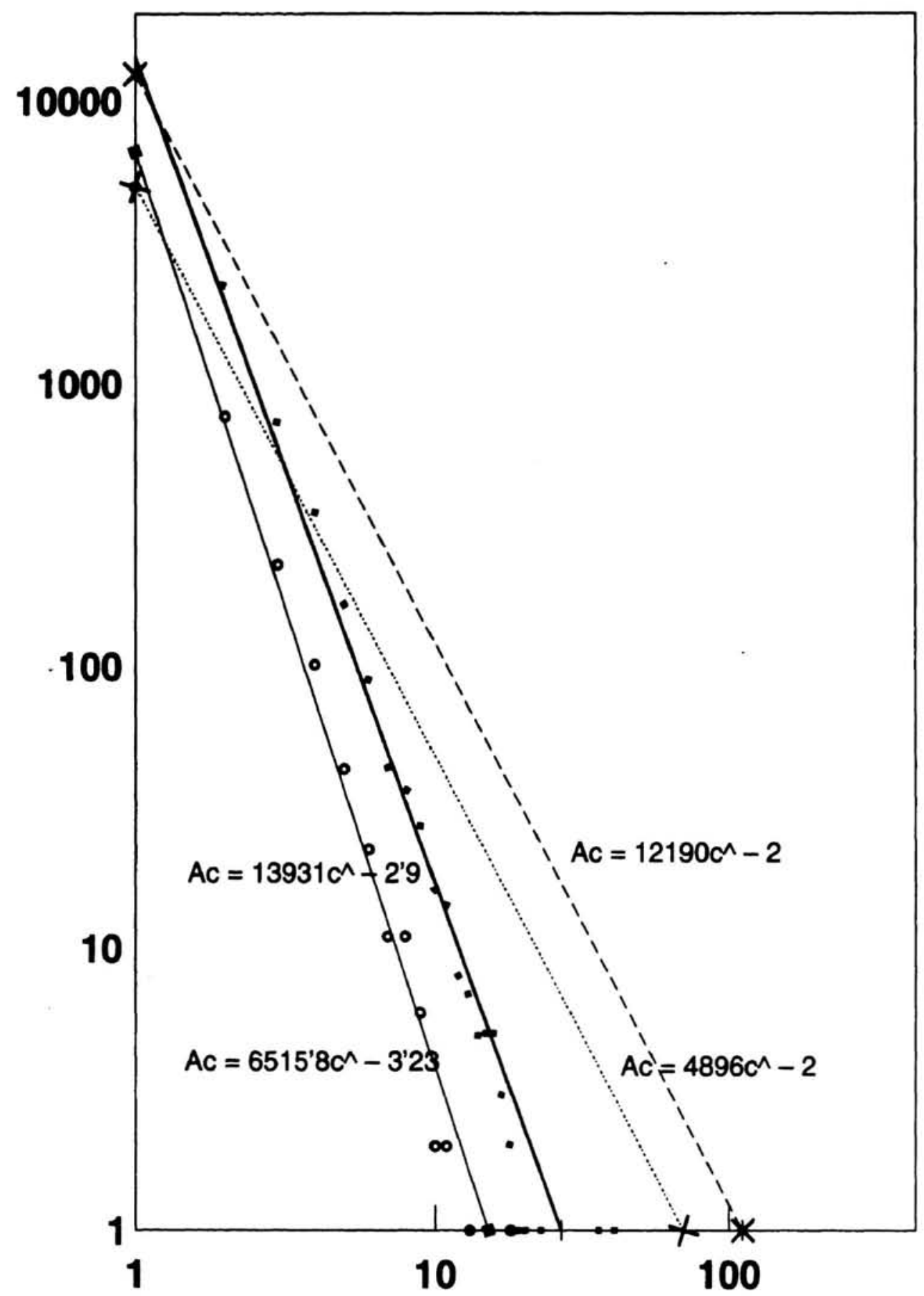




\section{Discusión}

La media obtenida de 2,87 autores por referencia proporciona una imagen que no se corresponde con la realidad que aportan otros estudios sobre la colaboración en la comunidad de autores médicos. López Piñero y Terrada (7) comprobaron que, en nuestro pars, el índice de firmas por trabajo ha pasado de 1,5 en 1968 a 3,5 en 1982. Desde esta fecha, y a pesar de algunas voces de alarma $(8,9)$ este indicador ha ido incrementándose, según estudios realizados en diversos campos. Así, por ejemplo, en 1984 la media de firmas por trabajo en los artículos originales publicados en la revista $\mathrm{Me}$ dicina Clínica (10) era de 5,6; en estudios más recientes de artículos publicados sobre neumología (11-16) se sitúa entre las cuatro firmas por trabajo en los artículos sobre asma bronquial (14) y las cinco de tumores de pulmón (12). En el mismo rango se sitúan los trabajos sobre radiodiagnóstico $(17,18)$ o sobre sida $(19)$. Este índice incluso aumenta en temas directamente relacionados con la cirugía (cinco en la revista Cirugía Española (20), seis en trasplantes (21)). El valor de 2,9 que hemos obtenido es inferior a todos los mencionados, menor que el de los estudios realizados sobre la literatura en patología digestiva (4,7 en patología gástrica (22), 5,2 en patología hepática (23)), menor al índice de firmas por trabajo de la literatura internacional $(7,10,24)$ e incluso menor a las 5,2 firmas por trabajo (25) que hemos calculado para los artículos publicados en la revista que analizamos y a las 5,7 que han calculado otros autores en la misma revista (26) para un período más corto.

Contribuyen a esta diferencia dos hechos: la alta influencia de la literatura internacional (25) cuyo índice de firmas por trabajo es inferior al de la literatura española, y sobre todo el que en las referencias analizadas no se relacionan todos los autores de los trabajos citados (en el $17,9 \%$ de las referencias puede observarse este problema), indicando en ocasiones menos de los que imponen las normas internacionalmente aceptadas (27). La exclusión en el cálculo de las referencias que contienen las partículas «et al» o «y col» tampoco ofrece una imagen ni siquiera aproximada de la realidad, pues en estas referencias están truncados precisamente los autores de los trabajos con mayor índice de coautoría.

La consecuencia es que, según se deduce del análisis de la bibliografía que presentamos, presumiblemente extensible a otras publicaciones médicas españolas, en tanto no se mantenga un control estricto sobre el cumplimiento de las normas aplicables a la referencia bibliográfica, el análisis de referencias, única vía para conocer los hábitos de colaboración en la literatura activa circulante en las distintas especialidades médicas, no será útil para este objetivo y habremos de conformarnos con analizar, como aproximación, la colaboración en los artículos publicados en un determinado período de tiempo, muchos de los cuales no tendrán influencia posterior en la comunidad científica.

El estudio realizado sobre los modelos de distribución de citas entre los autores (tabla 2, figura 1) muestra ciertas diferencias con respecto al modelo teórico previsto, en el sentido de un exceso de citas en el conjunto de los autores con escasa influencia (existe un exceso de autores con una o pocas citas), mientras que los autores con mayor influencia son menos de los esperados. Ello es, probablemente, consecuencia de la variedad de temas que se incluyen bajo el concepto de "cirugía digestiva», que precisa, en consecuencia, una gran variedad de fuentes de información, ampliando el número de publicaciones y de autores que son potencialmente citables y ello a pesar de que no se cita a todos los autores firmantes de las referencias. 
La diferencia entre la distribución teórica y la observada es aún mayor cuando se analiza la distribución de citas exclusivamente entre los primeros firmantes de cada una de las referencias. Ello puede explicarse en parte, además de por su interés, porque en múltiples ocasiones los trabajos son leídos y son citados porque entre sus firmantes se encuentran determinados profesionales cuya obra interesa a priori a los autores. Con gran frecuencia, sin embargo, estos profesionales de gran influencia firman sus trabajos en el segundo y sucesivos puestos en el orden de la firma $(20,23,28)$. Esta observación se confirma cuando se estudia la relación (tablas 3 y 4) de los primeros firmantes citados y de las citas a autores cuando no se tiene en cuenta el orden de la firma. Estas tablas muestran, en negrita, a los autores que figuran en ambas relaciones: sólo siete de los 20 autores más citados (con 15 o más citas) obtienen ocho o más citas como primeros firmantes; además, la diferencia entre el número total de citas obtenidas por cada autor y las que se deben a trabajos que firman en primer lugar es considerable en todos ellos.

Dos consecuencias se hacen evidentes: La primera que los repertorios de citas (como el conjunto de los que publica el ISI o la reciente experiencia del IME-Citas de Autores Médicos Españoles, que debe ser desarrollada y ampliada) son útiles para realizar estudios sobre el consumo de la información y un aspecto interesante de los mismos es el estudio de los autores. Sin embargo, puesto que hasta el momento no hacen referencia más que al primer firmante de cada uno de los trabajos citados, no son útiles para conocer a los autores más citados que, como queda demostrado, no siempre son los primeros firmantes de los trabajos. Las citas a los primeros firmantes pueden ser útiles para identificar los trabajos más citados, pero no a los autores que marcan las líneas y métodos de trabajo. Si estos repertorios pretenden ofrecer una imagen real de este último aspecto deben incluir, necesariamente, a todos los autores de los trabajos citados y no sólo a sus primeros firmantes.

La segunda consecuencia, en esta línea y confirmando nuestra hipótesis de partida, es que puede comprobarse la alta influencia que ejercen los autores españoles sobre nuestra propia literatura. El autor más citado es P. Parrilla, Catedrático de Cirugía de la Facultad de Medicina y Jefe del Departamento de Cirugía del Hospital Universitario Virgen de la Arrixaca de Murcia. Además, tres de los cinco autores más citados y seis de los 20 autores más citados son españoles. De los 20 autores más citados, sólo 7 no colaboran con el resto: se trata de Cameron, Pera Madrazo, Hogan, Morson, Thompson, De Meester y Dubois. El resto se organiza en cinco equipos de trabajo: el formado por Parrilla, Sánchez Bueno y Ramírez Romero; el que forman Starlz, Tzakis, Iwatsuki y Todo; en tercer lugar, García Alonso y Méndez; R. Williams forma equipo con Calne, y por último, Bloom y Polak. En definitiva, las máximas influencias proceden de 12 grupos de trabajo, de los cuales tres son españoles.

Por tanto, es fácil deducir que el uso que distintos organismos públicos españoles hacen de medidas de influencia o repercusión elaboradas en otras comunidades científicas (el muy conocido impact factor de Science Citation Index) para la evaluación de las publicaciones de autores españoles, sólo tiene sentido si, de forma consciente y aceptando las consecuencias que, entre otras, implícitamente pueden suponer acabar con la literatura científica en lengua española, se pretende que sus pautas de trabajo, temas y métodos imiten a los de la comunidad utilizada como referencia. En caso contrario, lo único que se consigue es un serio perjuicio para las revistas nacionales al forzar al investigador español a dirigir sus publicaciones a revistas extranjeras, funda- 
mentalmente norteamericanas y británicas y, en muchas ocasiones, a citar sus trabajos con el único fin de aumentar la probabilidad de aceptación del trabajo remitido. Si lo que se intenta es contar con un patrón de referencia con el que poder evaluar, de forma más o menos objetiva, las publicaciones españolas, como ya se ha apuntado (2), debe potenciarse la creación de repertorios e indicadores que tengan realmente en cuenta la producción nacional.

Por último, debemos señalar que el presente estudio ofrece todavía una visión limitada que deberá ser completada con el análisis no sólo de los autores, sino también de las publicaciones más influyentes, así como con estudios similares de otras publicaciones españolas en revistas nacionales, fundamentalmente en Cirugía Española que es la revista más utilizada por los especialistas en cirugía general para sus publicaciones en nuestro país $(20,29)$, y en revistas internacionales.

\section{Bibliografía}

1. BURTON, R. E.; KLEBER, R. N. The half-life of some scientific and technical literatures. Am Doc, 11:18-22, 1960.

2. LÓPEZ PIÑERO, J. M.; TERRADA, M. L. Los indicadores bibliométricos y la evaluación de la actividad cientifica. Med Clin (Barcelona), 98:101-106, 142-148, 384-388, 1992.

3. ORTEGA SERRANO, J.; MARTÍNEZ LÓN, J.; SALA PALAU, C. Autoboicot científico entre los cirujanos españoles: ¿Por qué no nos citamos? Cir Esp, 51:3-7, 1992.

4. HERNÁNDEZ VAQUERO, D. Las referencias bibliográficas en la «Revista de Ortopedia y Traumatología. Autoexclusión de los autores españoles. Revista de Ortopedia y Traumatologia, 36:529-35, 1992.

5. LOTKA, A. J. The frequency distribution of scientific productivity. J. Wash Acad Sci, 16:317, 1926.

6. PLATZ, A. Lotka's law and research visibility. Psychol Rep, 16:566-568, 1965.

7. LÓPEZ PIÑERO, J. M.; TERRADA, M. L. La información cientifica en medicina y sus fuentes. Valencia: Instituto de Estudios Documentales e Históricos sobre la Ciencia, 1993.

8. PÉREZ TRALLERO, E. Número de autores. ¿Cuál es el límite? Med Clin (Barcelona), 82(10): 470, 1984.

9. PULIDO, M. Los autores de los artículos médicos. Med Clin (Barcelona), 83(10):435, 1984.

10. CANELA SOLER, J.; OLLÉ GOIG, J. E. Muchos y bien avenidos: el número de autores en revistas clínicas. Med Clin (Barcelona), 89(14): 592-594, 1987.

11. GUTIÉRREZ GARCIA, J. Análisis bibliométrico de la producción cientifica española sobre oxigenoterapia (1973-1992) (tesis doctoral). Murcia: Dpto. Medicina Interna y Dpto. Historia de la Medicina, 1994.

12. EGEA PREFASI, C. Análisis de la producción científica española sobre tumores de pulmón (1980-1993) (tesis doctoral). Murcia: Dpto. Medicina Interna y Dpto. Historia de la Medicina, 1995.

13. GIMÉNEZ CORTÉS, M. E. Análisis de la producción cientifica española sobre neumonías infecciosas (1971-1993) (tesis doctoral). Murcia: Dpto. Medicina Interna y Dpto. Historia de la Medicina, 1995.

14. ROCA MEROÑO, M. Análisis bibliométrico de la producción científica española sobre asma bronquial (1974-1995) (tesis doctoral). Murcia: Dpto. Medicina Interna y Dpto. Historia de la Medicina; 1996.

15. BERNAL BELLIDO, C. Análisis bibliométrico de la producción científica española sobre enfermedad pulmonar obstructiva crónica (1971-1995) (tesis doctoral). Murcia: Dpto. Medicina Interna y Dpto. Historia de la Medicina, 1996. 
16. SERRA GOMEZ, C. Análisis bibliométrico de la producción cientifica española sobre tuberculasis (1985-1995) (tesis doctoral). Murcia: Dpto. Medicina Interna y Dpto. Historia de la Medicina, 1996.

17. MARTÍNEZ FERNÁNDEZ, M. Análisis bibliométrico de la producción cientffica espafiola sobre radiodiagnóstico a través de la revista «Radiologia» (1984-1993) (tesis doctoral). Murcia: Dpto. Radiología y Dpto. Historia de la Medicina, 1996.

18. MARTÍNEZ M.; SÁEZ, J. M.; GARCIA-MEDINA, V. Productividad de los autores espafoles en radiodiagnóstico. Análisis bibliométrico a través de la revista «Radiología (1984-1993). Radiología, 39(6):417-421, 1997.

19. LAJARIN BARQUERO, B. C. Análisis bibliométrico de la producción cientifica española sobre sida (1983-1991) (tesis doctoral). Murcia: Dpto. Medicina Interna y Dpto. Historia de la Medicina, 1994.

20. PARDO GARCIA, J. L. Análisis bibliométrico de la producción cientifica española sobre cirugía a través de la revista «Cirugía Española» (1974-1993) (tesis doctoral). Murcia: Dpto. Cirugía y Dpto. Historia de la Medicina, 1995.

21. CORTÉS GUARDIOLA, J. A. Un decenio de trasplantes: Análisis bibliométrico de la producción cientifica española sobre trasplantes de riñón, higado, corazón, pulmón y cardiopulmonar (1982-1991) (tesis doctoral). Murcia: Dpto. Medicina Interna y Dpto. Historia de la Medicina, 1994.

22. GÓMEZ ROIG, A. M. Análisis bibliométrico de la producción cientifica española sobre anatomía, fisiología y patología gástrica (1985-1994) (tesis doctoral). Murcia: Dpto. Medicina Interna y Dpto. Historia de la Medicina; 1996.

23. GIMÉNEZ VIUDES, J. Análisis bibliométrico de la producción científica española sobre patología hepática y de via biliar (1990-1996) (tesis doctoral). Murcia: Dpto. Medicina Interna y Dpto. Historia de la Medicina, 1997.

24. MARTÍN SÁRRAGA, F. O. Analisis de la producción cientifica en The Journal of Bone and Joint Surgery, edicion americana (1985-1994) (tesis doctoral). Murcia: Dpto. de Cirugfa y Dpto. Historia de la Medicina, 1997.

25. GOMEZ TERRADILLOS, D. Producción quirúrgica en «Revista Española de Enfermedades Digestivas», «Revista Quirúrgica Espaniola» y «Barcelona Quirúrgica» (1984-1995) (tesis doctoral). Murcia: Dpto. Cirugía y Dpto. Historia de la Medicina, 1997.

26. LÓPEZ-CÓZAR, E.; RUIZ-PÉREZ, R.; JIMÉNEZ-CONTRERAS, E. Calidad editorial, difusión e indicadores bibliométricos de la Revista Española de Enfermedades Digestivas. Rev Esp Enferm Dig, 91(1):1-16, 1999.

27. International Committee of Medical Journal Editors. Uniform requirements for manuscripts submitted to biomedical journals. $N$ Eng $J$ Med, 336:309-15, 1997.

28. GRECH RfOS, M. J. Análisis bibliométrico de la producción cientffico-médica en la Comunidad Autónoma de la Región de Murcia (1976-1994) (tesis doctoral). Murcia: Dpto. Historia de la Medicina y Dpto. Medicina Interna, 1996.

29. PARDO, J. L.; SÁEZ GÓMEZ, J. M.; DOMINGO GARCIA, P.; CARRASCO GONZÁLEZ, L.; ORTOLANO GOMEZ, A.; AGUAYO ALBASINI, J. L.; PARRILLA PARICIO, P. Importancia de «Cirugía Española» en el ámbito nacional de las publicaciones dedicadas a cirugía. Estudio bibliométrico en el período 1974-1993. Cirugía Española, 60(3): 207-212, 1996. 